\title{
Inpatient Cognitive Behavior Therapy Approaches for Suicide Prevention
}

Marjan Ghahramanlou-Holloway, Ph.D.

Laura L. Neely, Psy.D.

Jennifer Tucker, Ph.D.

Kathleen Caffery, B.A.

Victoria Colborn, B.S.

Viktor Koltko, B.S.

\author{
Address \\ "Department of Medical and Clinical Psychology, Laboratory for the Treatment of \\ Suicide-Related Ideation and Behavior, Uniformed Services University of the \\ Health Sciences, 4301 Jones Bridge Road, Room B3050, Bethesda, MD, 20814- \\ 4799, USA \\ Email: marjan.holloway@usuhs.edu
}

Published online: 14 October 2015

(C) Springer International Publishing AG (outside the USA) 2015

This article is part of the Topical Collection on Suicide

Keywords Cognitive behavior therapy - Inpatient - Attempted suicide - Psychiatric hospitalization - Suicide prevention

\section{Opinion Statement}

Within the current healthcare system, individuals recognized to be at imminent risk for suicide are likely to be referred for inpatient psychiatric care. The hospitalization setting provides a safe and supportive environment for specialty acute care services and stabilization. However, the majority of current interventions that are delivered to suicidal patients during this sensitive timeframe do not directly target suicide risk. Cognitive Behavior Therapy (CBT) is an evidence-based psychotherapeutic intervention that can easily be adapted for use in psychiatric inpatient settings. A specialized outpatient CBT protocol for suicide prevention has been developed and empirically evaluated by Drs. Aaron Beck and Gregory Brown at the University of Pennsylvania. This brief and targeted outpatient CBT program has been found to be efficacious in reducing the likelihood of subsequent suicide attempts by $50 \%$. Post-Admission Cognitive Therapy (PACT) is an inpatient adaptation of the CBT protocol for suicide prevention, developed by Dr. Marjan Ghahramanlou-Holloway at the Uniformed Services University of the Health Sciences. PACT is currently undergoing empirical validation, but it is important to note that the components of PACT are all cognitive behavioral practices recognized to reduce suicide risk. PACT is a promising inpatient CBT program that can be delivered in individual format to suicidal 
patients during their hospital stays. This intervention provides an opportunity to collaboratively review the suicide narrative, to conceptualize underdeveloped and overdeveloped skills directly tied to suicide risk, and to plan for safety and relapse prevention. Moreover, based on their conceptualization of the suicide mode activation for each patient, PACT clinicians choose cognitive behavioral strategies to modify thoughts, emotions, and/or behaviors that are most likely to result in a recurrence of suicidal behaviors. This paper provides a brief review of inpatient-based CBT options, introduces providers to the PACT inpatient protocol, and describes several promising alternate interventions.

\section{Introduction}

As you read this paper, consider that every 40 seconds, a person dies of suicide globally [1] and approximately every 13 minutes, a person dies of suicide in the United States of America (USA) [2]. Reliable estimates of suicide attempts globally and nationally are not available. However, it has been estimated that for every 25 suicide attempts, there is one suicide death, which translates to one person attempting suicide in the USA every 31 seconds [3]. The Substance Abuse and Mental Health Services Administration (SAMHSA) [4] reports that in 2013, 9.3 million American adults (3.9\%) experienced "serious thoughts of suicide in the past year" and 1.3 million American adults (0.6\%) attempted suicide.

During the same year, 494,169 individuals received services in US emergency departments due to selfinflicted injuries, although it is not clear if these injuries were intentional or unintentional [2]. Furthermore, 224,811 individuals were hospitalized for nonfatal selfinflicted injuries and 458,000 were estimated to have stayed at a hospital overnight or longer due to a suicide attempt $[2,4]$. The Agency for Healthcare Research and Quality (AHRQ) reports that the psychiatric length of stay for a principal diagnosis of suicide and intentional self-inflicted injury was 2.7 days with an estimated average cost of $\$ 11,851$ [5].
While it is clear that the psychiatric inpatient setting provides a safe and supportive environment for specialty acute care services and stabilization, questions about its efficacy in reducing suicide risk remain. According to a report commissioned by the Suicide Prevention Resource Center (SPRC) and SAMHSA [6], "[d]espite the centrality of hospitalizing seriously ill psychiatric patients, the research base for inpatient hospitalization for suicide risk is surprisingly weak. This review could not identify a single randomized trial about the effectiveness of hospitalization in reducing suicide acts after discharge" (p. 41). Moreover, well-powered randomized controlled trials (RCTs) that evaluate the efficacy of psychosocial interventions in reducing suicide risk among psychiatric inpatients are also lacking.

The broad objective of this paper is to review the current state of cognitive behavioral intervention research pertaining to the psychiatric inpatient setting and suicide prevention care. One promising cognitive behavioral intervention that has been specifically designed for delivery within an inpatient psychiatric setting and that directly targets the needs of suicidal patients is PostAdmission Cognitive Therapy (PACT) and will be reviewed in depth. Brief coverage about promising clinical approaches is provided with a discussion about next steps in advancing work in this important area.

\section{Inpatient cognitive behavior therapy approaches for suicide prevention}

Cognitive Behavior Therapy (CBT) is a form of psychotherapy that has been widely recognized as an evidence-based practice. The overwhelming majority of CBT studies have focused on its delivery in outpatient settings. Within the past two decades, CBT has been gaining increased usage within inpatient psychiatric 
settings and has been implemented for the treatment of a number of mental health conditions. For example, within the past 5 years alone, CBT inpatient studies have been published on the treatment of psychosis [7], anorexia nervosa [8], depression [9], and substance use disorders [10]. However, despite the increasing attention paid to inpatient cognitive behavioral interventions for a host of mental health conditions, research on inpatient CBT for individuals recently hospitalized following a suicide-related event remains limited.

Brown and Jager-Hyman [11•] state the following: "The dearth of knowledge about effective treatments for inpatient settings is especially alarming given that the current standard of care is to admit high-risk patients to inpatient units. This suggests that patients who are at high risk for suicide may not receive appropriate evidence-based treatments to prevent suicide" (p. S191). This gap in the scientific literature is probably due to a number of contributing factors. One factor may be related to the fact that historically, suicidal thoughts and behaviors have been treated in the context of the individual's psychiatric diagnosis and comorbidity. Nevertheless, the current consensus among CBT-driven suicidologists is that suicidal thoughts and behaviors must be targeted directly.

Cognitive behavioral treatment for suicide prevention is therefore viewed as transdiagnostic and adjunctive. This means that suicidal patients who receive CBT for suicide prevention during their inpatient stay are encouraged to engage in other forms of treatment that target the reduction of symptoms associated with their primary psychiatric diagnosis and comorbid conditions. For instance, a patient with the primary diagnosis of bipolar disorder II who is admitted for inpatient care due to a recent suicide attempt would be strongly advised to participate in evidence-based pharmacologic and psychosocial interventions designed for the treatment of the bipolar condition. In addition, this patient is a solid candidate to receive CBT for suicide prevention as a targeted intervention to reduce the likelihood of recurrent suicidal behaviors. Before providing an outline of inpatient CBT for suicide prevention, a brief review of the scientific literature pertaining to this topic is provided below.

\section{Review of cognitive behavior therapy research in inpatient settings}

A selection of evidence-based psychotherapies for suicide prevention is offered in a recent publication by Brown and Jager-Hyman [11•]. The authors present 16 studies, none of which involved the delivery of an inpatient intervention. The most comprehensive systematic review and meta-analysis on cognitive behavioral interventions for suicide prevention, to date, has been conducted by Nicholas Tarrier and colleagues and was published in 2008 [12••]. Studies that involved (a) at least one form of cognitive, behavioral, or cognitive behavioral intervention and a comparison condition and (b) any type of self-harm or suicidal behavior as an outcome measure were included in the meta-analysis. Of 8435 potential published articles since 1980, 28 studies met inclusion criteria. Only 3 out of the 28 studies delivered a full or partial intervention in an inpatient setting and these are described below. It is important to note that Keith Hawton and colleagues at the Center for Suicide Research at the 
Warneford Hospital Oxford have been working on an up-to-date systematic review of psychosocial studies for suicide prevention; the findings of this review are expected to be formally disseminated through the Cochrane Collaboration in the near future.

The first randomized controlled trial of a CBT inpatient intervention for suicide prevention was published in 1981 [13]. Twenty-four inpatients with a recent suicide attempt were randomized into either insight-oriented psychotherapy or behavior therapy groups. Patients in each group received 4 hours of therapy per day for approximately 1 week. Patients in both groups showed statistically significant improvements in depression, suicide ideation, and subsequent suicide attempts. However, behavior therapy patients showed statistically significant improvement when compared to insight-oriented therapy patients during the follow-up period. In Patsiokas and Clum's 1985 RCT [14], 15 inpatients admitted for a suicide attempt were randomized into cognitive restructuring, problem-solving, and non-directive (i.e., control) groups. Patients in each group received 10 individual therapy sessions over the course of 3 weeks. Regardless of treatment condition, all individuals showed improvements in hopelessness, suicide ideation, and suicide intent. Although these studies were underpowered, both demonstrated the potential utility of inpatient CBT programs for suicide prevention.

A third randomized controlled trial investigating an inpatient CBT approach to the prevention of suicide was published over a decade later [15]. In this study, 274 individuals who presented to an emergency department following a suicide attempt were randomized into treatment and control groups. Those in the treatment condition were admitted to a special crisis unit in the hospital where staff initiated discussions about the problems that led up to each individual's suicidal crisis. Following discharge, patients in the treatment condition were given 24-h emergency access to the special crisis unit, home visits as needed, and outpatient problem-solving treatment. Treatments for individuals in the control condition were determined by the emergency department clinicians (i.e., treatment as usual). It is interesting to note that only $25 \%$ of those in the control condition were psychiatrically hospitalized following their suicide attempts. Results of the study indicated no significant between-group differences on measures of hopelessness, symptomatology, or subsequent suicide attempts. In summary, while two of the three studies discussed above found no significant differences between CBT and control groups, methodological issues (including sample size, patient drop-out, and treatment packaging issues) made it difficult to ascertain the effects of the inpatient programs on suicide-related ideation and behavior.

In addition to the RCTs noted above, within the past decade, three clinical case studies have outlined the application of cognitive behavioral strategies for the inpatient care of suicidal individuals. The first case study [16] focused on the use of the cognitive model of depression [17] for treating those in an inpatient setting, but did not directly address the patient's suicidality. Two case studies with military service members psychiatrically admitted for suicide attempts have been published by PACT clinicians. The first case study [18] illustrated the feasibility of addressing a patient's suicidality in an inpatient setting and gave promising preliminary indications of PACT's effectiveness at addressing Beck's notion of the suicide mode [19] as a direct target for psychotherapy. While PACT is a suicide-focused therapy, clinical experience with PACT patients 
indicates that individuals with a diagnosis of posttraumatic stress disorder (PTSD) and/or extensive trauma histories can benefit from the individualized approach of establishing skills to cope with suicidal crises, which the third case study [20] illustrates. The use of PACT for a patient with multiple comorbid conditions demonstrates the importance of having a therapeutic technique which can address complex trauma while treating suicide risk.

\section{Post-Admission Cognitive Therapy for the prevention of suicide}

Given the dearth of evidence-based and efficacious treatments for suicide prevention in inpatient settings, the authors of this paper are currently engaged in the implementation of a well-powered, multisite randomized controlled trial of PACT among psychiatric inpatients at two military treatment facilities (with a regional Veterans Affairs Medical Center to be added as a third site). The research is supported by the Military Operational Medicine Research Program. PACT has been adapted from a 10-session efficacious outpatient CBT protocol for suicide prevention that has been shown to reduce subsequent suicide attempts by $50 \%$ in a community sample [21••] and by $60 \%$ in a military sample [22•]. The primary objective of the treatment is to reduce the likelihood of subsequent suicide attempts through the provision of a brief, adjunctive psychotherapeutic intervention.

Rather than viewing patients' problems through the lens of psychiatric diagnoses, PACT clinicians take a trans diagnostic, suicide-focused approach. The treatment group has included individuals with very high levels of trauma symptomatology, mild to moderate traumatic brain injury, substance and/or alcohol abuse or dependence, non-suicidal self-injury, maladaptive personality issues, and various forms of childhood and adulthood abuse, among other psychological and psychiatric disturbances. The underlying philosophy of the treatment is that while disturbances from various diagnoses may be related and in fact contribute to suicide risk either directly or indirectly, the suicidality of the individual deserves targeted attention. PACT clinicians are therefore trained to understand the specific role of psychopathology and life stressors in the activation, maintenance, and/or exacerbation of suicide risk. Clinicians guide conversations with patients in such a way that the focus remains on the patient's suicidality-its precipitating triggers, exacerbating factors, underlying causes-as much as possible during each session. This treatment is informed by the Aeschi approach to working with patients, which is characterized by the attitude that the patient is the expert of his/her experience and clinicians learn from patients how to best help them with their suicidality [23]. The Aeschi approach, similiar to cognitive behavior therapy, is a cooperative and collaborative therapeutic stance, rather than a prescriptive one.

The PACT inpatient treatment protocol is composed of four distinct phases: phase I-case conceptualization, phase II-skill acquisition, phase III-relapse prevention, and phase IV_outpatient aftercare. During phase I, patients are asked to tell their suicide narratives in as much detail as possible, in the form of a story with a beginning, middle, and end. This story, which is told at least twice, helps the patient and clinician understand the precipitating events, exacerbating factors, and overarching trajectory of a patient's suicidal crisis. The clinician uses this information to identify possible intervention points that could change the 
course of the crisis trajectory. In addition, during this phase, an initial safety plan is constructed collaboratively. This safety plan is targeted at keeping the patient safe during a potential suicidal crisis at the psychiatric inpatient unit. A safety plan is a hierarchically arranged list of warning signs and associated coping strategies to be used by the patient whenever a potential suicide-related situation arises. For more information on safety planning, see Stanley and Brown [24].

In phase II, the patient and clinician work together on skill-building tasks selected to ameliorate some of the deficits relevant to the onset, exacerbation, or perpetuation of the patient's suicidal thoughts, feelings, and behaviors. The clinician focuses on skills that are relevant to the patient's selected treatment goals, such as emotion regulation or decreasing feelings of loneliness and isolation. Activities used for skill acquisition include identifying and examining cognitive errors, reviewing and listing self-soothing experiences, and didactics pertaining to rational problem-solving strategies. The goal of this phase is to decrease putative risk factors for suicide.

For phase III, the patient and clinician work together to complete at least two relapse prevention exercises. Relapse in this context refers to a recurrence of suicidal self-directed violence, such as a suicide attempt. First, the dyad reviews the patient's previous suicide ideation and/or attempt narrative and inserts newly learned cognitive behavioral skills wherever appropriate and indicated. Next, the patient is asked to imagine a future scenario that could trigger suicidal behaviors. As the patient is telling the clinician the details of this scenario, the clinician stops the patient whenever appropriate and asks the patient what type of coping skill s/he could use to change the course of the suicide trajectory. The patient and clinician continue talking through the imagined scenario until the point of self-injury is imagined; the patient is then asked to name the ultimate steps that s/he could take for safety (e.g., calling 911 or going to a local emergency department). Through these exercises, the patient is given the opportunity to practice his/her newly acquired skills in an emotionally provocative yet safe situation.

Relapse prevention is ultimately aimed at helping patients gain confidence in their ability to cope effectively with a suicidal crisis that was previously overwhelming and confusing. Clinicians also inform patients that while suicidal thoughts may always be present in their lives, it is of the utmost importance that they refrain from acting on those thoughts. Patients are encouraged to use all of their available skills and resources to prevent a future suicide attempt. The final exercise of phase III is to review and update the patient's existing safety plan in order to prepare for psychiatric discharge and the patient's transition back to his or her life.

Phase IV of PACT takes place after the patient discharges from the hospital. It consists of four telephone booster sessions, ranging from 30 to 60 minutes in length, scheduled in the first 3 months post-discharge. During these sessions, the patient and clinician discuss his/her transition out of the hospital and back to work/school/home, his/her involvement with outpatient behavioral health treatment, and the appropriateness and helpfulness of the outpatient safety plan they developed. If the patient experiences difficulty in connecting with an outpatient provider, the clinician helps the patient identify any obstacles to treatment and then works collaboratively with the patient to identify potential solutions to the problem. This phase is aimed at ensuring continuity of care and 
helping patients transition to outpatient care. Throughout PACT, the clinician reminds the patient that PACT is considered an adjunctive treatment, to ideally take place alongside other concurrent treatments.

In summary, PACT is a short-term evidence-informed inpatient cognitive behavioral intervention focused on preventing future suicide attempts through skill and knowledge acquisition. PACT is distinguished from other therapeutic treatments by an intense focus on suicidality and its precipitants; the provision of therapy in an inpatient milieu; and emphases on skills acquisition, relapse prevention, and linkage to aftercare. While the safety and feasibility of PACT have been established in two pilot trials, data pertaining to the effectiveness of the intervention are still being collected. If PACT is found to be efficacious, the intervention can be subsequently disseminated to inpatient settings for those individuals at acute risk of suicidal behavior. For more information on PACT, see $[18,25 \bullet \bullet, 26]$. For more information on treating suicidal risk in inpatient settings, see $[27 \bullet \bullet]$.

The preliminary research and educational workshops on PACT have highlighted the need to further refine and tailor this intervention for wider dissemination across inpatient psychiatric settings. The ultimate goal is to integrate PACT more seamlessly into the existing inpatient milieu such that the intervention can be delivered more widely and efficiently without incurring additional costs and/or requiring more manpower, training, and resources within already stretched-to-the-limit inpatient systems of care. Hence, in addition to the individually delivered PACT protocol, a group therapy adaptation of PACT is currently in development for further refinement and pilot testing among psychiatric inpatients hospitalized due to suicide-related events.

While the PACT intervention is purely based on cognitive behavioral principles, a number of other interventions have been designed for the inpatient milieu and these utilize some aspects of CBT. These are reviewed in the section below.

\section{Additional promising clinical research for suicide prevention within inpatient settings}

\section{Collaborative Assessment and Management of Suicidality at Menninger}

David Jobes' Collaborative Assessment and Management of Suicidality (CAMS) [28] is a therapeutic framework that emphasizes collaborative assessment and treatment planning as a means of increasing therapeutic bond and motivation on behalf of the suicidal patient. While it is not a type of psychotherapy, CAMS has been adapted for use in inpatient settings $[29,30]$. The Collaborative Assessment and Management of Suicidality at Menninger (CAMS-M) approach, however, is intended to take on "more psychotherapeutic characteristics" [29] (p. 150) and includes elements from CBT for suicide prevention.

CAMS-M has been now examined in the context of a naturalistic, nonrandomized controlled-comparison trial consisting of 52 psychiatric inpatients [31]. Treatment was delivered in two 50-min individual psychotherapy sessions per week with the number of sessions ranging from 10 to 29, and a mean of approximately 15 sessions. Findings indicated significant improvement in two domains: suicide ideation and suicide-specific cognitions. Of note, 
the site of implementation for CAMS-M was the Menninger Clinic, which has an average length of stay of 59 days. This should be compared with the national average length of psychiatric inpatient stays-approximately 3 days [32]. Therefore, the generalizability of these CAMS-M findings to other psychiatric inpatient settings may be limited and must be examined in future studies.

\section{Dialectical Behavior Therapy}

Dialectical Behavior Therapy (DBT), an evidence-based treatment, was developed by Marsha Linehan [33] for female outpatients diagnosed with borderline personality disorder. The first randomized controlled trial of outpatient DBT [34] showed a significant reduction in the length of psychiatric inpatient hospitalization days. Results of DBT adaptations for use in an inpatient setting have been mixed. "Initial empirical studies that attempted to modify DBT for use on adult inpatient units (these modifications included features not consistent with DBT principles) produced equivocal results and raised questions about the effectiveness of this treatment approach for inpatients" [34] (p. 277).

In 2000, Bohus and colleagues [35] reported that a pilot study of 24 female inpatients treated with DBT showed a reduction of suicidal behaviors, anxiety, stress, depression, and dissociation. The inpatient DBT consisted of 3 months of treatment followed by a transition to long-term outpatient treatment. The first phase of DBT involved 3 weeks of behavior analysis, the second phase involved theoretical training on condition, skills acquisition, and contingency management, and the third phase focused on discharge planning. In 2004, Katz and colleagues [36] reported on a 2-week DBT inpatient adolescent program that was adapted from the 12-week outpatient adolescent program [37]. Treatment consisted of individual DBT sessions twice per week, 10 daily manualized DBT skills training sessions, and DBT milieu for skills generalization. Adolescents in the DBT inpatient adolescent milieu showed significant reductions in behavioral incidents when compared to adolescents receiving standard treatment. However, at the 1-year follow-up, there were no differences between the groups on suicidal thoughts and behaviors or depressive symptoms. In summary, while DBT clearly shows promise for the treatment of suicidal individuals (particularly those with borderline personality disorder), additional research is needed to best understand the adaptability of this long-term intervention for adult inpatient settings.

\section{Other inpatient psychosocial interventions under investigation}

In order to find currently unpublished studies of inpatient CBT for suicide prevention, we conducted a search of the National Institutes of Health RePORTER database for the terms "inpatient AND suicide" in the project title, project terms, and project abstract. The search encompassed the years 20052015 and yielded a total of 85 studies. Several of the studies found were notable for their innovative inpatient interventions.

1. Dr. David Brent at the University of Pittsburgh and Dr. Beth Kennard at the University of Texas Southwestern Medical Center are examining a manualized intervention that includes a phone app to assist with the transition of suicidal adolescents from inpatient to outpatient care. The intervention, called ASAP, consists of "(1) Adherence-promoting engagement and adherence to treatment through motivational 
interviewing; (2) Safety planning; and (3) Affect Protection-selecting from a menu of techniques for maintaining positive affect."

2. Dr. Peter Britton at the Syracuse Veterans Affairs Medical Center is conducting a trial to evaluate a two-session inpatient administration of Motivational Interviewing to address Suicidal Ideation (MI-SI) that includes a one telephone booster session after discharge. The study population is US military veterans.

3. Dr. Paul Pfeiffer at the University of Michigan is evaluating an inpatient peer support program that addresses two suicide-related risk factors-hopelessness and social isolation.

4. Dr. Shirley Yen at Brown University is conducting treatment development research to evaluate the Coping Long-term with Attempted Suicide Program-Adolescents (CLASP-A). The intervention consists of three individual meetings with the adolescent during the inpatient stay, one in-person meeting with the patient and his or her parent/guardian during the inpatient stay, and a number of scheduled telephone calls with the adolescent and his or her parent/guardian during the 6-month post-discharge period.

In addition to the NIH-funded studies noted above, O'Connor and colleagues [38] have published on their pilot trial of Teachable Moment Brief Intervention (TMBI) for medically admitted suicide attempt survivors. The TMBI was developed and tested as an innovative protocol, focusing on a brief window of time following the suicide attempt for those medically hospitalized. A total of 30 patients were randomized to receive either TMBI or treatment as usual. TMBI aims to provide a onetime intervention that can be implemented on acute inpatient medical units while taking into account the challenges of this setting. The authors maintain that the brief amount of time after an impactful event is ideal for an intervention because the individual is more open and has more motivation to change behaviors. TMBI incorporates aspects of DBT as well as CAMS. The provider meets with the patient and helps him or her identify the events leading up to the suicide attempt. In addition, there is an effort to examine the impact of the attempt on different aspects of the suicide attempt survivor's life. The provider and the patient collaboratively complete a document recording these factors and a plan to respond to them in future situations. Since TMBI consists of only one meeting (averaging approximately $44 \mathrm{~min}$ ), it has the potential to be implemented in a number of different inpatient medical settings without disrupting the standard of care already being provided. Study findings demonstrated that those randomized to TMBI in comparison to the control condition showed improvements in reasons for living, readiness to change, and motivation to address problems.

For patients hospitalized for suicide-related reasons, the inpatient milieu provides a unique window of opportunity to reflect upon their reasons 


\section{Acknowledgments}

for dying versus their reasons for living. The examination of the chain of events that ultimately led to one's suicidal thoughts and/or behaviors cannot and should not be carried out by the patient alone. The inpatient hospital stay presents the first opportunity to effectively involve a suicidal patient in treatment aimed to engage him or her in guided discovery, to build hope for the future, to practice skills for managing subsequent suicidal crises (e.g., safety planning and relapse prevention), to enhance overall motivation to live, and to prepare for linkage to aftercare following psychiatric discharge. Cognitive behavior therapy for suicide prevention is one form of psychotherapy that has proven to be effective in targeting and reducing suicide risk in outpatient settings. This efficacious treatment has now been adapted for inpatient use in the context of Post-Admission Cognitive Therapy. Inpatient clinicians are encouraged to learn more about the cognitive behavioral components of PACT and other promising inpatient-based psychotherapies. Researchers are urged to develop and empirically evaluate additional psychotherapy interventions-including variations of CBT-that can be feasibly delivered within inpatient psychiatric settings either in individual and/or group formats.

Funding support for the preparation of this manuscript has been provided to Principal Investigator, Dr. Ghahramanlou-Holloway, by the Department of Defense, Congressionally Directed Medical Research Program (W81XWH-082-0172), Military Operational Medicine Research Program (W81XWH-11-20106), and the National Alliance for Research on Schizophrenia and Depression (15219).

\section{Compliance with ethics guidelines}

\section{Conflict of Interest}

Marjan Ghahramanlou-Holloway declares that he has no conflict of interest.

Laura Neely declares that she has no conflict of interest.

Jennifer Tucker declares that she has no conflict of interest.

Kathleen Caffery declares that she has no conflict of interest.

Victoria Colborn declares that she has no conflict of interest.

Viktor Koltko declares that he has no conflict of interest.

\section{Human and animal rights and informed consent}

This article does not present animal and/or human subjects data but references previous research with human subjects conducted by one or more of the authors (references $18,20,25 \bullet \bullet, 26,27 \bullet \bullet$ ). These prior studies were approved and overseen by the appropriate Institutional Review Boards. All participants provided written informed consent prior to enrollment. 


\section{Disclaimer}

The views expressed in this manuscript are those of the authors and do not necessarily reflect the official policy or position of the Department of Defense or the US Government.

\section{References and Recommended Reading}

Papers of particular interest, published recently, have been

highlighted as:

- Of importance

- Of major importance

1. Preventing suicide: a global imperative [Internet]. World Health Organization: Geneva, 2014. ISBN 978 924156477 9. Available from: http://www.who.int/ mental_health/suicide-prevention/world_report_ 2014/en/.

2. Centers for Disease Control and Prevention (CDC) - Web-based Injury Statistics Query and Reporting System (WISQARS) [Internet]. National Center for Injury Prevention and Control, CDC (producer), 2013. Available from: www.cdc.gov/injury/ wisqars/index.html.

3. Drapeau CW, McIntosh JL (for the American Association of Suicidology) [Internet]. U.S.A. suicide 2013: official final data. Washington, DC: American Association of Suicidology, 2015. Available from http://www. suicidology.org.

4. Substance Abuse and Mental Health Services Administration (SAMHSA), Center for Behavioral Health Statistics and Quality. The NSDUH report: substance use and mental health estimates from the 2013 national survey on drug use and health: overview of findings. Rockville, MD; 2014.

5. Weiss AJ (Truven Health Analytics), Barrett ML (M.L. Barrett, Inc.), Steiner CA (AHRQ). Trends and projections in inpatient hospital costs and utilization, 20032013 (HCUP Statistical Brief \#175). Agency for Healthcare Research and Quality, Rockville, MD; 2014. Available from http://www.hcup-us.ahrq.gov/reports/ statbriefs/sb175-Hospital-Cost-Utilization-

Projections-2013.pdf.

6. Knesper DJ, American Association of Suicidology, \& Suicide Prevention Resource Center. Continuity of care for suicide prevention and research: suicide attempts and suicide deaths subsequent to discharge from the emergency department or psychiatry inpatient unit. Newton: Education Development Center, Inc.; 2010.

7. Owen M, Sellwood W, Kan S, Murray J, Sarsam M. Group CBT for psychosis: a longitudinal, controlled trial with inpatients. Behav Res Ther. 2015;65:76-85.

8. Grave R, Calugi S, Conti M, Doll H, Fairburn C. Inpatient cognitive behaviour therapy for anorexia nervosa: a randomized controlled trial. Psychother Psychosom. 2013;82:390-8. doi:10.1159/000350058.

9. Forkmann T, Scherer A, Pawelzik M, et al. Does cognitive behavior therapy alter emotion regulation in inpatients with a depressive disorder? Psychol Res Behav Manag. 2014;7:147-53. doi:10.2147/PRBM. S59421.

10. Van Dam D, Ehring T, Vedel E, Emmelkamp PMG. Trauma-focused treatment for posttraumatic stress disorder combined with CBT for severe substance use disorder: a randomized controlled trial. BMC Psychiatry. 2013;13:172. doi:10.1186/1471-244X-13-172.

11. Brown GK, Jager-Hyman S. Evidence-based psychotherapies for suicide prevention: future directions. Am J Prev Med. 2014;47:S186.

This article provides a discussion of evidence-based psychotherapy approaches for suicide prevention and a series of recommendations for future research.

12.• Tarrier N, Taylor K, Gooding P. Cognitive-behavioral interventions to reduce suicide behavior: a systematic review and meta-analysis. Behav Modif. 2008;32:77-108.

This article provides a detailed meta-analytic review of cognitive behavioral interventions for suicide prevention.

13. Liberman RP, Eckman T. Behavior therapy vs insightoriented therapy for repeated suicide attempters. Arch Gen Psychiatry. 1981;38:1126.

14. Patsiokas AT, Clum GA. Effects of psychotherapeutic strategies in the treatment of suicide attempters. Psychotherapy. 1985;22:281-90.

15. Van der Sande R, van Rooijen L, Buskens E, et al. Intensive in-patient and community intervention versus routine care after attempted suicide. A randomised controlled intervention study. Br J Psychiatry. 1997;171:35-41.

16. Abramovich E. Application of CBT in an inpatient setting: case illustration of an adult male with anxiety, depression, and Axis II symptoms. Clin Case Stud. 2006;5:305-30.

17. Beck AT, Emery G. Anxiety disorders and phobias: a cognitive perspective. New York: Basic Books; 1985.

18. Neely LL, Irwin K, Ponce JTC, Perera K, Grammer G, Ghahramanlou-Holloway M. Post-Admission Cognitive Therapy (PACT) for the prevention of suicide in military personnel with histories of trauma: treatment development and case example. Clin Case Stud. 2013;12:457-73.

19. Beck AT. Beyond belief: a theory of modes, personality, and psychotherapy. In: Salkovaskis PM, editor. 
Frontiers of cognitive therapy. New York: Guilford; 1996. p. 1-25.

20. Tucker J, Armstrong K, Neely LL, GhahramanlouHolloway M. The treatment of trauma-related suicidal behavior: expanding the knowledge base with a focused case study. In: Martin C, Preedy VR, Patel VB, editors. The comprehensive guide to post-traumatic stress disorder. New York: Springer; 2015.

21.• Brown GK, Ten Have T, Henriques GR, Xie SX, Hollander JE, Beck AT. Cognitive therapy for the prevention of suicide attempts: a randomized controlled trial. JAMA. 2005;294(5):563-70.

This article reports on the efficacy of a brief outpatient cognitive behavioral intervention in reducing the likelihood of future suicide attempts.

22.• Rudd MD, Bryan CJ, Wertenberger EG, et al. Brief cognitive-behavioral therapy effects on post-treatment suicide attempts in a military sample: results of a randomized clinical trial with 2-year follow-up. Am J Psychiatry. 2015;172:441-9.

This article provides data to support the adaptation of cognitive behavior therapy for suicide prevention for military service members receiving outpatient mental health services.

23. Jobes DA, Ballard E. The therapist and the suicidal patient. In: Michel K, Jobes DA, editors. Building a therapeutic alliance with the suicidal patient. Washington: American Psychological Association; 2011. p. 51-61.

24. Stanley B, Brown GK. Safety planning intervention: a brief intervention to mitigate suicide risk. Cogn Behav Pract. 2012;2011(19):256-64.

25.• Ghahramanlou-Holloway M, Cox DW, Greene FN. Post-Admission Cognitive Therapy: a brief intervention for psychiatric inpatients admitted after a suicide attempt. Cogn Behav Pract. 2012;19:233-44.

This article describes the components of an inpatient cognitive behavioral intervention, titled, Post-Admission Cognitive Therapy (PACT).

26. Ghahramanlou-Holloway M, Neely LL, Tucker J. A cognitive behavioral strategy for preventing suicide. Curr Psychiatr. 2014;13(8):18-28.

27.• Ghahramanlou-Holloway M, Neely LL, Tucker J. Treating risk for self-directed violence in inpatient settings. In: Bryan CJ, editor. A guide to brief cognitive behavioral treatments for suicide risk across clinical settings. New York: Routledge; 2015.

This book chapter focuses on treatment options for suicidal patients admitted for inpatient psychiatric care.

28. Jobes DA. Managing suicidal risk. New York: Guilford; 2006.

29. Ellis T, Daza P, Allen J. Collaborative Assessment and Management of Suicidality at Menninger (CAMS-M): an inpatient adaptation and implementation. Bull Menn Clin. 2012;76:147-71.

30. Ellis TE, Green KL, Allen JG, Jobes DA, Nadorff MR. Collaborative Assessment and Management of Suicidality in an inpatient setting: results of a pilot study. Psychotherapy. 2012;49:72-80.

31. Ellis TE, Rufino KA, Allen JG, Fowler JC, Jobes DA. Impact of a suicide-specific intervention within inpatient psychiatric care: the Collaborative Assessment and Management of Suicidality. Suicide Life Threat Behav. 2015.

32. Healthcare Cost and Utilization Project (HCUP). HCUP Nationwide inpatient sample (NIS). Rockville: Agency for Healthcare Research and Quality; 2010.

33. Linehan MM. Cognitive-behavioral treatment of borderline personality disorder. New York: Guilford; 1993.

34. Linehan MM, Armstrong HE, Suarez A, Allmon D, Heard HL. Cognitive-behavioral treatment of chronically parasuicidal borderline patients. Arch Gen Psychiatry. 1991;48:1060-4.

35. Bohus M, Haaf B, Stiglmayr C, Pohl U, BoÈhme R, Linehan M. Evaluation of inpatient DialecticalBehavioral Therapy for borderline personality disorder-a prospective study. Behav Res Ther. 2000; 875-887.

36. Katz LY, Cox BJ, Gunasekara S, Miller AL. Feasibility of Dialectical Behavior Therapy for suicidal adolescent inpatients. J Am Acad Child Adolesc Psychiatry. 2004;43:276.

37. Miller AL, Rathus JH, Linehan M, Wetzler S, Leigh E. Dialectical Behavior Therapy adapted for suicidal adolescents. J Pract Psychiatry Behav Health. 1997;3:7886.

38. O'Connor SS, Comtois KA, Wang J, et al. The development and implementation of a brief intervention for medically admitted suicide attempt survivors. Gen Hosp Psychiatry. 2015. 